\title{
Electrokinetically Modified Water
}

National Cancer Institute

\section{Source}

National Cancer Institute. Electrokinetically Modified Water. NCI Thesaurus. Code

C117733.

Orally available, nanobubble-based, electrokinetically modified water (EMW) composed of reverse osmosis water where the minerals calcium chloride, magnesium chloride, and potassium bicarbonate have been added and with an increased oxygen concentration compared to normal water. Upon consumption, the EMW may have a beneficial effect on fatigue. This water may protect muscle cells against damage and may improve skeletal muscle function. The water is pretreated with strong, controlled turbulence to create charge-stabilized nanostructures. 